\title{
Laparoscopic and robotic ureteral stenosis repair: a multi- institutional experience with a long-term follow-up
}

\author{
Riccardo Schiavina $^{1} \cdot$ Stefano Zaramella $^{2} \cdot$ Francesco Chessa $^{1} \cdot$ Cristian Vincenzo Pultrone $^{1}$. \\ Marco Borghesi ${ }^{1} \cdot$ Andrea Minervini $^{3} \cdot$ Andrea Cocci $^{3} \cdot$ Andrea Chindemi $^{3} \cdot$ Alessandro Antonelli $^{4}$. \\ Claudio Simeone $^{4} \cdot$ Vincenzo Pagliarulo $^{5} \cdot$ Paolo Parma $^{6} \cdot$ Alessanrdo Samuelli $^{6}$. \\ Antonio Celia ${ }^{7}$ - Bernardino De Concilio ${ }^{7}$ - Bernardo Rocco ${ }^{8}$ - Elisa De Lorenzis ${ }^{8}$. \\ Gaetano La Manna $^{9} \cdot$ Carlo Terrone $^{2} \cdot$ Mario Falsaperla $^{10} \cdot$ Donato Dente $^{11} \cdot$ Angelo Porreca $^{11}$
}

Received: 1 December 2015/ Accepted: 26 April 2016

(C) Springer-Verlag London 2016

\begin{abstract}
The treatment of ureteral strictures represents a challenge due to the variability of aetiology, site and extension of the stricture; it ranges from an end-to-end anastomosis or reimplantation into the bladder with a Boari flap or Psoas Hitch. Traditionally, these procedures have been done using an open access, but minimally invasive approaches have gained acceptance. The aim of this study is to evaluate the safety and feasibility and perioperative results of minimally invasive surgery for the treatment of ureteral stenosis with a long-term follow-up. Data of 62 laparoscopic $(n=36)$ and robotic $(n=26)$ treatments for ureteral stenosis in 9 Italian centers were reviewed. Patients were followed according to the referring center's protocol. Laparoscopic and robotic approaches were compared. All the procedures were completed successfully without open conversion. Average estimated blood loss in the two groups was $91.2 \pm 71.9 \mathrm{cc}$ for the laparoscopic and $47.2 \pm 32.3 \mathrm{cc}$ for the robotic, respectively $(p=0.004)$. Mean days of hospitalization were $5.9 \pm 2.4$ for the laparoscopic group and $7.6 \pm 3.4$ for the robotic group $(p=0.006)$.
\end{abstract}

Francesco Chessa

francesco.chessa2@studio.unibo.it

1 Department of Urology, University of Bologna, S. OrsolaMalpighi Hospital, Palagi 9 Street, 40134 Bologna, Italy

2 Department Of Urology, University of Eastern Piedmont Azienda Ospedaliero-Universitaria Maggiore Della Carità, Novara, Italy

3 Department of Urology, Careggi Hospital, University of Florence, Florence, Italy

4 Department of Urology, University of Brescia, Brescia, Italy

5 Department of Urology, University of Bari “Aldo Moro", Bari, Italy
No differences were found in terms of operative time and post-operative complications. After a median followup of 27 months, the robotic group yielded 2 stenosis recurrence, instead the laparoscopic group shows no cases of recurrence $(p=0.091)$. Minimally invasive approach for ureteral stenosis is safe and feasible. Both robotic and pure laparoscopic approaches may offer good results in terms of perioperative outcomes, low incidence of complications and recurrence.

Keywords Robotics · Laparoscopy · Ureter · Stenosis · Ureteral obstruction - Reconstructive surgical procedures

\section{Introduction}

Ureteral stenosis may result from a variety of causes including stone passage, endoscopic procedures, endometriosis, urothelial carcinoma, radiation therapy, previous open or laparoscopic surgery and penetrating injuries [1]. Ureteroscopy with lithotripsy represents one of

6 Department of Urology, Ospedale "Carlo Poma" Mantova, Mantova, Italy

7 Dept. Of Urology, Ospedale "San Bassiano", Bassano del Grappa, VI, Italy

8 Department of Urology, Policlinico Di Milano, Milan, Italy

9 Department of Experimental, Diagnostic and Specialty Medicine, University of Bologna, S. Orsola-Malpighi Hospital, Bologna, Italy

10 Department of Urology, Policlinico Vittorio Emanuele, Catania, Italy

11 Department of Urology, Policlinico Di Abano, Abano Terme, PD, Italy 
the main causes; ureteral strictures in fact occur as a late complication in $0-4 \%$ of cases [2]. Endometriosis rarely involves the upper urinary tract, $(1-2 \%$ of all cases of stenosis), $84 \%$ of which are associated with concomitant bladder involvement $[3,4]$. The incidence of iatrogenic ureteral injury ranges between $0.1 \%$ and $2.5 \%$ of all the surgical procedures $[5,6]$; during recent years, an increasing incidence of iatrogenic strictures has been observed, due to the widespread diffusion of abdominal and gynaecological laparoscopic surgery and upper urinary tract endoscopy [7].

Treatment for ureteral stenosis ranges from a conservative endoscopic dilatation to surgical approach with end-to-end anastomosis or reimplantation of the ureter in the bladder with or without a Boari flap or Psoas Hitch. The surgical strategy is based on site, length and aetiology of the ureteral stenosis. For benign, proximal and mid-ureteral stenosis $<5 \mathrm{~mm}$, an initial endoscopic management is indicated. Proximal stenosis shorter than 3-4 $\mathrm{cm}$ can usually be managed with a primary end-toend anastomosis [8]; conversely for proximal strictures $>4 \mathrm{~cm}$ in length, the interposition of an ileal segment can be necessary. [9]. Distal ureteral stenosis is usually managed by ureteral reimplantation: when the ureter is too short for direct reimplantation, additional length can be gained by fixation of the bladder to the tendon of the psoas muscle (the psoas hitch suspension), necessitating a wide mobilization of the bladder to perform a tension-free anastomosis; in case of very long damage of the distal ureter, a tubulization of the bladder to gain the iliac/lumbar ureter is required (the Boari flap) [10].Traditionally, all these procedures were being done using an open approach [11], but with the recent widespread use of minimally invasive surgery, laparoscopic and robot-assisted approaches have been proposed even for the treatment of ureteral stenosis.

Open surgical procedures show good long-term results, but are usually associated with long hospitalization, need of additional pain medication and greater blood loss [12]. Furthermore, the open approach has many cosmetic consequences, especially in young patients and females. Laparoscopic and robot-assisted procedures have been introduced as alternatives to open surgery with the aim to reduce morbidity and hospitalization. Several studies [1012] have demonstrated the feasibility and safety of minimally invasive procedures; however, to our knowledge, only few studies have reported robotic and pure laparoscopic approach with long-term follow-up.

The aim of the present study is to evaluate the feasibility of laparoscopic or robotic ureteral repair for stenosis due to different aetiology at different level and with long-term follow-up.

\section{Patients and methods}

In this multicentre retrospective study, we collected patients who underwent robotic or laparoscopic surgery for ureteral stenosis between March 2008 to March 2014, at 9 Italian centers with long-term experience in minimally invasive urological surgery. This series included ureteral stenosis for different aetiologies and different location in the ureter, excluding primary uretero-pelvic junction obstruction.

Before surgery, all patients underwent computed tomography (CT) to evaluate the site and the extent of the stricture. The following pre-operative variables were collected: age, gender, body mass index (BMI), comorbidities classified according to Charlson Index. Stenosis were classified by side, site, length and aetiologies. The following operative parameters were analysed: surgical approach, surgical technique, operative time, estimated blood loss and intra-operative complications. Post-operative complications were graded with the Clavien-Dindo classification [13].

Follow-up examinations were done at the discretion of the referring center. All patients were followed up with clinical examination, blood exams with serum creatinine measurement and renal ultrasound or CT at 3-6 months and with regular imaging evaluation thereafter. A CT scan and diuretic $99 \mathrm{mTc}$-mercaptoacetyltriglycine (MAG3) renography were performed in cases of suspected restenosis and hydronephrosis.

\section{Surgical technique}

\section{End-to-end anastomosis}

In cases of proximal and mid-ureteral stenosis $\leq 3-4 \mathrm{~cm}$ in length, uretero-ureteroanastomosis was performed. Although in the distal stenosis, the treatment of choice was the ureteral reimplantation; in 10 cases, surgeons preferred the uretero-ureteroanastomosis technique. During a transperitoneal approach, the mobilization of the colon in the left side and the kocherization of the second and third portion of the duodenum in the right side were done. The ureter was mobilized, preserving the adventitia and the area immediately proximal and distal to the stricture was excised; an interrupted or continuous anastomosis with $4-0$ or $5-0$ monocryl was performed with a double $\mathrm{j}$ stent into the ureter.

\section{Ureteral reimplantation}

In cases of distal ureteral stenosis $<20 \mathrm{~mm}$ in length, an ureteral reimplantation was performed. After the isolation of the ureter, a cystotomy was made for the reimplantation 
of the ureter. Prior to complete the ureteroneocystostomy with $4 / 0$ interrupted stitches, a double $\mathrm{J}$ stent was placed anterogradely. Then, the cystotomy was closed with 3-0 absorbable suture. At the end of the intervention, a Foley catheter is placed for 5 days.

\section{Psoas hitch reimplantation}

In case of distal ureteral stenosis, to obtain a tension-free anastomosis, a Psoas Hitch reimplantation represent the option of choice The Psoas Hitch reimplantation is an alternative technique used in case of longer distal stenosis providing a tension-free anastomosis. [10]. The ureter was dissected preserving its blood supply. The bladder was mobilized from the anterior abdominal wall by incising the peritoneum laterally. The contralateral umbilical artery can be sectioned to improve its mobilization. The Psoas muscle was exposed to hitch the bladder and 2-3 sutures are placed to tie the bladder to the psoas muscle, superficially. The ureter was spatulated and anchored to the bladder previously dissected. The anastomosis is done according to the surgeon's preferences A double $\mathbf{J}$ stent was placed in an anterograde approach and finally the bladder is closed in two layers with continuous suture.

\section{Post-operative care}

A Jackson-Pratt o Penrose drain was left in all of these procedures; the drain was removed on post-operative day 1-3. The Foley catheter was removed after the drain was removed. The ureteral stent was removed around 3-4 weeks post-operatively using flexible or rigid cystoscope.

\section{Statistical analysis}

Mean (SD), range, and frequencies were used as descriptive statistics. The Mann-Whitney, Pearson 2, linear-bylinear association, and Fisher exact tests were used as appropriate. Statistical analyses were conducted using SPSS version 17.0 (IBM Corp, Armonk, NY, USA). Twotailed $p$ values less than 0.05 were considered statistically significant.

\section{Results}

The demographics and pre-operative clinical characteristics of the patients are summarized in Table 1. In 14 patients, stenosis was localized in the lumbar ureter, in 3 cases in the iliac ureter and in 45 patients distally in the pelvic ureter. In the present series, ureteral stenosis was due to different causes: endometriosis (30 patients),
Table 1 Patient demographics (no. 62)

\begin{tabular}{|c|c|}
\hline Variables & Value \\
\hline \multicolumn{2}{|l|}{ Age in years } \\
\hline Mean \pm DS & $44.6 \pm 13.8$ \\
\hline Median (IQR) & $44.0(35-52)$ \\
\hline \multicolumn{2}{|l|}{ Gender, $n(\%)$} \\
\hline Male & $12(19.4)$ \\
\hline Female & $50(80.6)$ \\
\hline \multicolumn{2}{|l|}{ BMI } \\
\hline Median (IQR) & $25.1(22-28)$ \\
\hline \multicolumn{2}{|l|}{ Charlson index*, $n(\%)$} \\
\hline 0 & $28(46)$ \\
\hline 1 & $10(16)$ \\
\hline 2 & $11(17)$ \\
\hline 3 & $8(13)$ \\
\hline 4 & $4(6)$ \\
\hline 6 & $1(2)$ \\
\hline \multicolumn{2}{|l|}{ Side of the stenosis, $n(\%)$} \\
\hline Right & $28(45.2)$ \\
\hline Left & $34(54.8)$ \\
\hline \multicolumn{2}{|l|}{ Site of the stenosis, $n(\%)$} \\
\hline Lumbar & $14(22.6)$ \\
\hline Iliac & $3(4.8)$ \\
\hline Pelvic & $45(72.6)$ \\
\hline \multicolumn{2}{|l|}{ Aetiology, $n(\%)$} \\
\hline After URS** & $15(24.2)$ \\
\hline After abdominal surgery & $11(17.7)$ \\
\hline Endometriosis & $30(48.4)$ \\
\hline Other & $6(9.7)$ \\
\hline \multicolumn{2}{|c|}{ Lumbar ureteral stenosis length $(n=14), \mathrm{mm}$} \\
\hline Mean \pm DS & $20.4 \pm 0.8$ \\
\hline Median (IQR) & $20.0(13-26)$ \\
\hline \multicolumn{2}{|c|}{ Iliac ureteral stenosis length $(n=3), \mathrm{mm}$} \\
\hline Mean \pm DS & $15.7 \pm 0.9$ \\
\hline Median (IQR) & $15.0(0.7-15)$ \\
\hline \multicolumn{2}{|c|}{ Pelvic ureteral stenosis length $(n=45), \mathrm{mm}$} \\
\hline Mean \pm DS & $25.2 \pm 1.1$ \\
\hline Median (IQR) & $22.2(20-30)$ \\
\hline \multicolumn{2}{|c|}{ Pre-operative serum creatinine } \\
\hline Mean \pm DS & $1.18 \pm 0.42$ \\
\hline Median (IQR) & $1.1(0.8-1.5)$ \\
\hline
\end{tabular}

iatrogenic such as ureteroscopy with lithotripsy (15 patients) or abdominal surgery (11 patients). In 5 cases, ureteral stenosis was due to idiopathic or post-irradiation retroperitoneal fibrosis and in 1 patient due to urinary tuberculosis. The mean ureteral stenosis length was $23.6 \mathrm{~mm} \pm 1.1$. All the procedures were completed 
Table 2 Operative and post-operative outcomes

\begin{tabular}{ll}
\hline Variables & Value \\
\hline Previous treatment & \\
Stent placement & $29(46)$ \\
Balloon dilatation & $6(10)$ \\
Surgical Approach, $n(\%)$ & \\
Laparoscopic & $36(58)$ \\
Robotic & $26(42)$ \\
Retroperitoneal approach & $11(17)$ \\
Surgical technique, $n(\%)$ & \\
Uretero-ureteroanastomosis & $27(43.5)$ \\
Ureteral reimplantation & $22(35.5)$ \\
Psoas Hitch Reimplantation & $13(21.0)$ \\
Operative time (min) & \\
Mean \pm DS & $173 \pm 106$ \\
Median (IQR) & $145(90-210)$ \\
Estimated blood loss (cc) & \\
Mean \pm DS & $73.2 \pm 62.5$ \\
Median (IQR) & $50(40-90)$ \\
Post-operative serum creatinine & \\
Mean \pm DS & $25.6 \pm 16.1$ \\
Median (IQR) & $1.0 \pm 0.3$ \\
Length of hospitalization, (days) & $1.0(0.8-1.2)$ \\
Mean \pm DS & \\
Median (IQR) & $6.6 \pm 2.9$ \\
Stent placement, (days) & \\
Mean \pm DS & $31.4 \pm 16.5$ \\
Median (IQR) & $24.5-31.5)$ \\
Stenosis recurrence, $n(\%)$ & \\
Follow-up (month) & \\
Mean \pm DS & \\
Median (IQR) & \\
\hline
\end{tabular}

successfully without open conversion. The operative and post-operative outcomes are reported in Table 2; in 35 cases $(56 \%)$, a conservative approach with retrograde double $\mathrm{J}$ stent placement or balloon dilatation was attempted before surgery. Overall, 36 patients were treated with pure laparoscopic approach and 26 with robot-assisted surgery with the Da Vinci SI robotic system (Intuitive Surgical, Sunnyvale, CA, United States). In 11 cases, a retroperitoneal laparoscopic approach was preferred to repair a proximal/iliac ureteral stenosis. Short strictures $(<25 \mathrm{~mm})$ in the proximal or distal ureter were treated with end-to-end anastomosis. Patients with longer distal strictures underwent a direct ureteral reimplantation or a Psoas Hitch reimplantation. Uretero-ureterostomy were performed in 27 patients, ureteral reimplantation in 22 patients and a Psoas Hitch reimplantation was performed in 13 patients. After a median follow-up of 27 months, a recurrence of the stenosis was diagnosed in 2 cases: one case required a balloon dilatation, one case required a laser endoureterotomy; both cases resolve completely. Table 3 shows the comparison between laparoscopic and robotic approach: robotic approach had some advantages compared to pure laparoscopic surgery in terms of estimated blood loss. On the other hand, the laparoscopic approach resulted in fewer days of hospitalization. No significant differences were found in terms of operative time, stenosis recurrence, renal function and complications rate between the two groups. Table 4 shows the type and the grade of intraoperative and post-operative complications observed in the present series according to the Clavien-Dindo classification. Post-operative complications $\leq 90$ days after surgery occurred in $5(8 \%)$ patients. The most frequent complication was prolonged fever (Clavien 1) in 2 patients, Anaemia (Clavien 2) due to haematuria that required blood transfusion in 1 patients, and prolonged ileus (Clavien 1) in one case. In only 1 case, a major complication occurred: an ureteral fistula (Clavien 3B) during robot-assisted ureteroureteroanastomosis for deep infiltrating endometriosis, who required the placement of additional nephrostomy.

\section{Discussion}

The surgical management of ureteral strictures is a complex challenge for the urologist; it depends mainly on the location, length and aetiology of the stenosis. In addition to the endoscopic management with stent placement or balloon dilatation, surgical approach may be performed by traditional open surgery or by laparoscopic or robot-assisted approach.

Nezhat et al. first described the first laparoscopic uretero-ureterostomy for ureteral stenosis due to endometriosis in 1992 [14]. Laparoscopic ureteroneocystostomy in children was first described by Ehrlich [15] and the first in adult was reported by Reddy and Evans in 1994 [16]. With increasing experience and expertise, the minimally invasive approach for ureteral reconstructive procedures such as uretero-ureteroanastomosis or ureteral reimplantation with Psoas Hitch has gained acceptance [17-19].

However, laparoscopic approach for ureteral stenosis is considered a complex procedure due to difficulties in intracorporeal suturing, two-dimensional vision and the limited movement of the instruments [10]. Modi et al. reported their experience describing the results of 6 patients who underwent laparoscopic ureteroneocystostomy. They concluded that an experienced laparoscopic surgeon and good dexterity with intracorporeal laparoscopic suturing technique are essential for a successful completion of the procedure [18]. Similarly, De Cicco et al., in a review of 608 ureteral injuries treated with endoscopic, open and laparoscopic surgery, concluded that 
Table 3 Comparison between laparoscopic and robotic groups

\begin{tabular}{|c|c|c|c|}
\hline Variables & Laparoscopic $(n=36)$ & Robotic $(n=26)$ & $P$ value \\
\hline \multicolumn{4}{|l|}{ Age } \\
\hline Mean \pm DS & $48.4 \pm 14.0$ & $39.4 \pm 12.0$ & \multirow[t]{2}{*}{0.011} \\
\hline Median (IQR) & $48.0(40.7-58.5)$ & $37.0(32.5-46.2)$ & \\
\hline \multicolumn{4}{|l|}{ Gender, $n(\%)$} \\
\hline Male & $8(22.2)$ & $4(15.3)$ & \multirow[t]{2}{*}{0.501} \\
\hline Female & $28(77.8)$ & $22(84.7)$ & \\
\hline \multicolumn{4}{|l|}{ BMI } \\
\hline Mean \pm DS & $25.7 \pm 3.7$ & $23.4 \pm 4.0$ & \multirow[t]{2}{*}{0.194} \\
\hline Median (range) & $25.6(23-28)$ & $23.4(19.9-25.9)$ & \\
\hline \multicolumn{4}{|l|}{ Side of the stenosis, $n(\%)$} \\
\hline Right & $17(47.2)$ & $11(57.6)$ & \multirow[t]{2}{*}{0.701} \\
\hline Left & $19(52.8)$ & $15(42.4)$ & \\
\hline \multicolumn{4}{|l|}{ Site of the stenosis, $n(\%)$} \\
\hline Lumbar & $12(33.3)$ & $2(7.7)$ & \multirow[t]{3}{*}{0.049} \\
\hline Iliac & $2(5.5)$ & $1(3.9)$ & \\
\hline Pelvic & $22(61.2)$ & $23(88.4)$ & \\
\hline \multicolumn{4}{|l|}{ Aetiology, $n(\%)$} \\
\hline After URS** & $12(33.3)$ & $3(11.5)$ & \multirow[t]{4}{*}{0.058} \\
\hline After abdominal surgery & $7(19.4)$ & $4(15.1)$ & \\
\hline Endometriosis & $12(33.3)$ & $18(70.2)$ & \\
\hline Other & $5(14.0)$ & $1(3.2)$ & \\
\hline \multicolumn{4}{|l|}{ Lumbar ureteral stenosis length, $\mathrm{mm}$} \\
\hline Overall & 12 & 2 & \multirow[t]{3}{*}{0.088} \\
\hline Mean \pm DS & $18.8 \pm 0.71$ & $30.0 \pm 1.4$ & \\
\hline Median (IQR) & $20.0(11-23)$ & $30.0(20-30)$ & \\
\hline \multicolumn{4}{|l|}{ Iliac ureteral stenosis length, $\mathrm{mm}$} \\
\hline Overall & 2 & 1 & \multirow[t]{3}{*}{0.347} \\
\hline Mean \pm DS & $20.0 \pm 0.7$ & 0.7 & \\
\hline Median (IQR) & $20.0(15-20)$ & $0.7(0.7)$ & \\
\hline \multicolumn{4}{|l|}{ Pelvic ureteral stenosis length, $\mathrm{mm}$} \\
\hline Overall & 22 & 23 & \multirow[t]{3}{*}{0.444} \\
\hline Mean \pm DS & $22.6 \pm 1.0$ & $23.9 \pm 1.3$ & \\
\hline Median (IQR) & $25.0(25-30)$ & $20.0(10-31)$ & \\
\hline Retroperitoneal access n (\%) & $4(11)$ & $7(26)$ & 0.757 \\
\hline \multicolumn{4}{|l|}{ Surgical technique, $n(\%)$} \\
\hline End-to-end anastomosis & $20(55.5)$ & $5(19.2)$ & \multirow[t]{3}{*}{0.081} \\
\hline Ureteral reimplantation & $10(27.8)$ & $9(34.6)$ & \\
\hline Psoas Hitch Reimplantation & $6(16.7)$ & $3(11.5)$ & \\
\hline \multicolumn{4}{|l|}{ Operative time $(\min )^{* * *}$} \\
\hline Mean \pm DS & $163.0 \pm 102.5$ & $185 \pm 112.0$ & \multirow[t]{2}{*}{0.440} \\
\hline Median (IQR) & $130.0(100-197)$ & $199.0(80.0-222.0)$ & \\
\hline Serum Creatinine variation, $\mathrm{mg} / \mathrm{dL}$ & $-0.17 \pm 0.2$ & $-0.09 \pm 0.3$ & 0.089 \\
\hline & $-0.1(-0.3$ to 0$)$ & $-0.1(-0.2$ to 0$)$ & \\
\hline Estimated blood loss (cc) & & & \\
\hline Mean $\pm \mathrm{DS}$ & $91.2 \pm 71.9$ & $47.2 \pm 32.3$ & 0.004 \\
\hline Median (IQR) & $50.0(50-115)$ & $50.0(30.0-50.0)$ & \\
\hline Length of hospitalization, (days) & & & \\
\hline Mean \pm DS & $5.9 \pm 2.4$ & $7.6 \pm 3.4$ & 0.006 \\
\hline Median (Range) & $5.0(4.0-.0)$ & $7.0(6.0-8.0)$ & \\
\hline
\end{tabular}


Table 3 continued

\begin{tabular}{llll}
\hline Variables & Laparoscopic $(n=36)$ & Robotic $(n=26)$ & $P$ value \\
\hline Stent placement, (days) & & & \\
$\quad$ Mean \pm DS & $31.3 \pm 12.7$ & $31.5 \pm 21.1$ & 0.509 \\
$\quad$ Median (IQR) & $30.0(25-30)$ & $29.0(21-35)$ & \\
Stenosis recurrence, $n(\%)$ & $1(2.7)$ & & 0.091 \\
Follow-up (month) & & $26.4 \pm 12.1$ & 0.100 \\
Mean \pm DS & $28.7 \pm 14.4$ & $23.0(13.5-28.5)$ & \\
Median (IQR) & $25.0(18-37)$ & $2(3.2)$ & 0.395 \\
Clavien complications, $n(\%)$ & $1(1.6)$ & 0 & \\
Grade 1 & $1(1.6)$ & $1(1.6)$ & \\
Grade 2 & 0 & & \\
Grade 3 & & & \\
\hline Bold & & & \\
\hline
\end{tabular}

Bold values indicate $\mathrm{p}$ value $<0.05$

* Charlson index with age

** Ureterorenoscopy with lithotripsy

*** Console time for robotic approach

Table 4 Type of post-operative complications

\begin{tabular}{llll}
\hline Cases, $n(\%)$ & Complication & Treatment & Dindo/Clavien system \\
\hline \multicolumn{2}{l}{ Post-operative complications } & & \\
$1(1.6)$ & Ureteral Fistula & Nephrostomy + stent & Grade 3B \\
$1(1.6)$ & Anaemia & Medical therapy & Grade 2 \\
$1(1.6)$ & Ileus & Medical therapy & Grade 1 \\
$2(3.2)$ & Prolonged Fever & Medical therapy & Grade 1 \\
\hline
\end{tabular}

laparoscopy represents the method of choice for the management of ureteral lesions, but requires specialized centers and experienced laparoscopic surgeons due to the technical difficulty of the anastomosis of such a small structure as the ureter [20].

The introduction of the Da Vinci Robot has reduced the technical difficulties of laparoscopy, especially during the reconstructive phases. Furthermore, several studies have shown that the learning curve of the robotic-assisted surgery is easier than the laparoscopic one. Passerotti et al. reported that among inexperienced surgeons, the efficiency of suturing using Robot-assisted surgery was operator-independent and required less time to learn [21]. Likewise, Yohannes and Jacobs showed that robotic surgery reduces the learning curve for experienced open surgeons when beginning with robotic approach, compared to conventional laparoscopy [22, 23].

The first robot-assisted ureteroneocystostomy was described in 2003 by Yohannes et al. [24], and the first robot-assisted reimplantation with Psoas hitch was reported by Naeyer et al. in 2007 [25]; both the authors concluded that robot-assisted reimplantation could be performed easier and with greater technical precision than conventional laparoscopy.
So far, there are few multicentres, large-sample series with long-term follow-up reporting the safety and feasibility of robotic ureteral reconstruction.

Musch et al. in a study of 16 patients showed that robotassisted reconstructive surgery of the distal ureter is feasible without compromising the generally accepted principles of open surgical procedures [26].

Patil et al. in a multinstitutional study analysed 12 patients who underwent robot-assisted ureteric reimplantation with Psoas Hitch. They concluded that ureteral reimplantation can be performed safely and effectively with a robotic-assisted laparoscopic technique and is associated with excellent outcomes with minimal post-operative morbidity [10].

Hemal et al. analysed 44 patients who underwent robotassisted surgeries for different ureteral pathologies with a mean follow-up of 13.5 months. They confirmed that robot-assisted surgery could be successfully used for surgical management of most ureteral pathologies including complex ureteral reconstruction [11].

Our experience comprises one of the largest series in the literature considering laparoscopic and robotic surgery for ureteral strictures due to several aetiologies and with various reconstruction technique. Only very few studies, 
to our knowledge, have reported a comparison between robotic and laparoscopic approach regarding ureteral surgery, most of them concerning pyeloplasty for ureteropelvic junction obstruction $[27,28]$. Lucas et al. analysed data from 759 patients who underwent laparoscopic or robotic pyeloplasty (274 laparoscopic, 465 robotic), and found less estimated blood loss in the laparoscopic group. No differences in terms of intra-operative and post-operative complications were found between the two groups [27]. Similarly, Baldie et al. in a comparative study between robotic and laparoscopic surgery for distal ureteral stenosis showed less estimated blood loss and less operative time in the laparoscopic group [28]. In our study, we showed a statistically significant difference in terms of estimated blood loss in favour of robotic group. The laparoscopic group showed shorter hospitalization time; however, the latter data could be also influenced by the different behaviour among the study centers; there was no difference considering all the other endpoints. As already reported in other studies comparing laparoscopic and robotic surgery, the rate of estimated blood loss and days of hospitalization are variable and often depend on the experience of the surgeon in laparoscopic and robotic techniques [29, 30]. Most of the stenosis in our cohort involved the distal ureter. In the pelvis, the ureteral reconstruction may be technically challenging when the dissection is extensive because the vascular supply to the distal ureter may be compromised. This can potentially cause the recurrence of strictures, the most common complication reported in the literature [31]. However, in our series, we report a low rate of recurrence with only 2 cases of stenosis recurrence.

Similarly to other studies, the overall rate of complications was low $(8 \%)[11,12,27]$ with only 1 high grade (Clavien III) complication that occurred in a patients who underwent robotic uretero-ureteroanastomosis for deep infiltrating endometriosis.

This study, to our knowledge, represents one of the few that compares laparoscopic and robotic approach for ureteral strictures, with a long-term follow-up.

This study presents some limitations: its retrospective design and, as a multi-institutional study, there were differences in surgical technique among surgeon of each institution enrolled in the study.

\section{Conclusion}

The present retrospective series has shown that laparoscopic and robot-assisted management of ureteral stenosis, due to various aetiologies, is feasible and safe. After a long-term follow-up, the functional outcomes were optimal with a low incidence of complications and low rate of recurrence of the stenosis. Minimally invasive treatment of ureteral stenosis should be taken into account in skilled centers.

\section{Compliance with ethical standards}

All patients provided informed consent for anonymous publication of data.

Conflict of interest Riccardo Schiavina, Stafano Zaramella, Francesco Chessa, Cristian Vincenzo Pultrone, Marco Borghesi, Andrea Minervini, Andrea Cocci, Andrea Chindemi, Alessandro Antonelli, Claudio Simeone, Vincenzo Pagliarulo, Paolo Parma, Alessandro Samuelli, Antonio Celia, Bernardino De Concilio, Bernardo Rocco, Elisa De Lorenzis, Carlo Terrone, Gaetano La Manna, Mario Falsaperla, Donato Dente and Angelo Porreca declare that they have not conflict of interest.

Ethical approval The study was done in accordance with the Helsinki declaration and national regulations.

Informed consent All patients provided informed consent for anonymous publication of data.

\section{References}

1. Nakada SY, Hsu TH (2012) Management of upper urinary tract obstruction. In: Kavoussi LR, Novick AC, Partin AW, Peters CA (eds) Cambpell-Walsh urology, 10th edn. Elsevier Saunders, Philadelphia, pp 1122-1168

2. Johnson DB, Pearle MS (2004) Complications of ureteroscopy. Urol Clin North m 31:157-171

3. Westney OL, Amundsen CL, McGuire EJ (2000) Bladder endometriosis: conservative management. J Urol 163(6):1814-1817

4. Antonelli A, Simeone C, Zani D et al (2006) Clinical aspects and surgical treatment of urinary tract endometriosis: our experience with 31 cases. Eur Urol 49(6):1093-1097

5. Selzman AA, Spirnak JP (1996) Iatrogenic ureteral injuries: a 20-year experience in treating 165 injuries. J Urol 155:878-881

6. Gangai MP, Agee RE, Spence CR (1976) Surgical injury to ureter. Urology 8:22-27

7. Parpala-Sparman T, Paananen I, Santala M et al (2008) Increasing numbers of ureteric injuries after the introduction of laparoscopic surgery. Scand J Urol Nephrol 42:422-427

8. Hsu THS, Streem SB, Nakada SY (2007) Management of upper urinary tract obstruction. In: Wein AJ, editor. Campbell-Walsh Urology. ed 9.Philadelphia, PA: WB Saunders

9. Wagner JR, SchimpfMO Cohen JL (2008) Robot-assisted laparoscopic ileal ureter. JSLS 12:306-309

10. Patil Nilesh N, Mottrie Alexandre, Sundaram Bala, Vipul R (2008) Patel robotic-assisted laparoscopic ureteral reimplantation with psoas hitch: a multi-institutional, multinational evaluation. Urology 72:47-50

11. Hemal Ashok K, Nayyar Rishi, Gupta Narmada P, Lalgudi N (2010) Dorairajan experience with robot assisted laparoscopic surgery for upper and lower benign and malignant ureteral pathologies. Urology 76:1387-1394

12. Rassweiller JJ, Gozev AS, Erdogru T (2007) Ureteral reimplantation for management of ureteral strictures: a retrospective comparison of laparoscopic and open techniques. Eur Urol 51:512-522

13. Clavien PA, Barkun J, De Oliveira ML et al (2009) The Clavien-Dindo classificationof surgical complications: five-year experience. Ann Surg 250:187-196 
14. Nezhat C, Nezhat F, Seidman D et al (1992) Laparoscopic repair of ureter resected during operative laparoscopy. Obset Gynecol 80:543-544

15. Ehrlich RM, Gershman A, Fuchs G (1994) Laparoscopic vesicouretero-plasty in children: initial case reports. Urology 43:255

16. Reddy PK, Evans RM (1994) Laparoscopic ureteroneocystostomy. J Urol 152(6 pt 1):2057-2059

17. Chung H, Jeong BC, Kim HH (2006) Laparoscopic ureteroneocystostomy with vesicopsoas hitch: nonrefluxing ureteral reimplantation using cystoscopy-assisted submucosal tunneling. J Endourol 20:632-638

18. Modi P, Goel R, Dodiya S (2005) Laparoscopic ureteroneocystostomy for distal ureteral injuries. Urology 66:751-753

19. Inderbir S. Gill, Stephen J. Savage, Anthony J (2000) Laparoscopic Ileal Ureter The Journal Of Urology 163:1199-1202

20. De Cicco C, Ret Dávalos ML, Van Cleynenbreugel B (2007) Iatrogenic ureteral lesions and repair: a review for gynecologists. J Minim Invasive Gynecol 14:428-435

21. Passerotti CC, Passerotti AM, Dall'Oglio MF et al (2009) Comparing the quality of the suture anastomosis and the learning curves associated with performing open, freehand, and roboticassisted laparoscopic pyeloplasty in a swine animal model. J Am Coll Surg 208:576-586

22. Yohannes P, Rotariu P, Pinto P et al (2002) Comparison of robotic versus laparoscopic skills: is there a difference in the learning curve? Urology 60:39-45

23. Bruce L. Jacobs, Samuel R. Kaufman, Hal Morgenstern, Trends In The Treatment Of Adults With Ureteropelvic Junction Obstruction Journal Of Endourology Volume 27, Number 3, March 2013
24. Yohannes P, Chiou RK, Pelinkovic D (2003) Rapid communication: pure robot-assisted laparoscopic ureteral reimplantation for ureteral stricture disease: case report. J Endourol 17:891-893

25. Naeyer GD, Migem PV, Schatteman P et al (2007) Case report: pure robot-assisted psoas hitch ureteral reimplantation for distalureteral stenosis. J Endourol 21:618-620

26. Musch Michael, Hohenhorst Lukas (2013) Anne Pailliart Robotassisted reconstructive surgery of the distal ureter: single institution experience in 16 patients. BJU Int 111:773-783

27. Steven M. Lucas, Chandru P. Sundaram (2012) Factors that impact the outcome of minimally invasive pyeloplasty: results of the multi-institutional laparoscopic and robotic pyeloplasty collaborative group. J Urol 187(2):522-527

28. Baldie K, Angell J, Ogan K et al (2012) Robotic management of benign mid and distal ureteral strictures and comparison with laparoscopic approaches at a single institution. J Urol 80(3):596-601

29. Rozet F, Harmon J, Cathelineau X, Barret E, Vallancien G (2006) Robot-assisted versus pure laparoscopic radical prostatectomy. World J of Urol 24(2):171-179

30. Fonseka T, Ahmed K, Froghi S, Khan SA, Dasgupta P, Shamim Khan M (2015) Comparing robotic, laparoscopic and open cystectomy: a systematic review and meta-analysis. Arch Ital Urol Androl 87(1):41-48

31. Phillips EA, Wang DS (2012) Current status of robot-assisted laparoscopic ureteral reimplantation and reconstruction. Curr Urol Rep 13(3):190-194 